\title{
Modulation of Instantaneous Synchrony During Seizures by Deep Brain Stimulation
}

\author{
Ananda S. Fine \\ Department of Bioengineering \\ University of Illinois, Chicago
}

\author{
David P. Nicholls \\ Department of Mathematics, Statistics \\ and Computer Science \\ University of Illinois, Chicago
}

\author{
David J. Mogul \\ Department of Biomedical Engineering \\ Illinois Institute of Technology
}

\begin{abstract}
Epileptic seizures were experimentally induced in the CA3 region of rat hippocampus in vivo. Recordings of seizure activity were made in both hippocampi as well as anteromedial region of the thalamus in order to analyze the instantaneous activity for synchronous oscillators. A new method is introduced for detecting this synchrony which combines empirical mode decomposition, the Hilbert analytic signal method and eigenvalue decomposition. Effects of targeted deep brain stimulation on multi-site synchrony were assessed as a means to extinguish hypersynchrony during epileptic seizures.
\end{abstract}

Keywords - empirical mode decomposition; synchrony; epilepsy

\section{INTRODUCTION}

Synchronous oscillatory activity has been found to be a critical component in both normal brain states such as binding of visual information [1] as well as pathological states such as Parkinson's disease [2] [3], schizophrenia [4], Alzheimer's disease [5] and epilepsy [6] [7]. However, detecting synchronization behavior in the brain represents an extraordinarily difficult problem in neurological science. Although various statistics have been proposed for the detection of phase synchrony from multiple electrodes placed at varying degrees of neuronal resolution (i.e. depth, subdural and/or surface), many of these methods rely on several assumptions that render them inappropriate for detecting synchrony in the brain. All electrophysiological signals besides single cell recordings are a summation of activity from areas surrounding the electrode. In particular, local field potentials (LFPs) represent a sum of dendritic activity that may be inhibitory or excitatory (inhibitory or excitatory postsynaptic potentials, respectively). Thus, these signals represent multiple components leading to a necessity for filtering. Most often, filtering of these signals involves either clinically determined ranges (e.g. alpha, beta, etc.) or Fourier spectrum derived bandwidths. Although these bandwidths determined a priori may, in many cases, yield useful narrow band signals, it would be more advantageous for a filtering algorithm to make no assumptions as to the underlying components of the signal under study.

An ideal analysis method would be capable of extracting proper waveforms adaptively from the time series without any a priori assumptions and without the need to build complicated mathematical models derived from first principles (a daunting task for modeling dynamics beyond anything but a small population of neurons). A relatively recent technique combining well-known results in linear algebra and mean-field theory has been proposed to obtain significant synchrony clusters within bivariate phase data measures. This method, termed the "eigenvalue decomposition method", utilizes directional statistical features of the phase dynamics between any two oscillatory signals to define significantly synchronized clusters of oscillators [8] [9]. This type of analysis relies on an assumption that there are several mean fields of globally coupled phase oscillators within the signal set. This approach figures prominently in our new analysis. We present here an analytic process applied to intracranial EEG information recorded in multiple deep brain nuclei bilaterally in the rat brain that merges the techniques of empirical mode decomposition, the Hilbert analytic signal method, mean phase coherence measures and finally eigenvalue decomposition to ultimately identify complex instantaneous synchronous behavior. Such a procedure may provide important new insights as a seizure or any other complex neurological process in the brain evolves in time and space.

\section{METHODS}

\section{A. Surgical Procedure/Data Acquisition}

Male Sprague-Dawley rats, 48-57 days old and weighing approximately 225-280 gm were used in this study. Experiments were conducted in accordance with the National Institutes of Health for the care and use of laboratory animals. Rats were anesthetized by a mixture of Ketamine $(70 \mathrm{mg} / \mathrm{kg})$ and Xylazine $(2 \mathrm{mg} / \mathrm{kg})$ delivered intraperitoneally. All procedures were performed in a Kopf stereotactic frame (KOPF Model 900, CA, USA). Stereotactic targets were calculated using a stereotactic rat brain atlas [10]. The skull was perforated using a high speed stereotactic drill (Micromotor ${ }^{\mathrm{TM}}$ Drill, Stoelting Co, IL USA) with $1.2-2 \mathrm{~mm}$ diameter drill tips. Bipolar electrodes surrounding a single stainless steel injection cannula in one integrated electrode assembly (C315G-MS 303: PlasticsOne, Roanoke, VA, USA) were stereotactically implanted into the CA3 region of the left hippocampus (-3.5 mm Bregma, $2.8 \mathrm{~mm}$ lateral, $3.7 \mathrm{~mm}$ deep). Bipolar recording electrodes (without cannula) were implanted into the contralateral hippocampus and anteromedial thalamus (-1.8 $\mathrm{mm}$ Bregma, $0.3 \mathrm{~mm}$ lateral, 6.1 $\mathrm{mm}$ deep). After injection of epileptogenic chemicals into the $\mathrm{CA} 3$ region of the left hippocampus, the internal cannula 
insert was withdrawn and a stainless steel insert was threaded through the cannula to provide one side of the recording pair. We used a chemical induction model of epilepsy in order to induce a physiological state that has been classically described as "hypersynchronous". Each experiment involved recording one half hour of baseline activity followed by focal injection of $3-5$ nmol kainic acid into the CA3 region of the left hippocampus.

\section{B. Empirical Mode Decomposition}

Traditional spectral decompositions applied to time series usually involve an assumption that the underlying signal dynamics consist of a linear superposition of complex exponentials. However, these radial basis functions are always assumed a priori rather than obtained adaptively from the signal. If the signal of interest contains more than just pure sine and cosine functions, the resulting power spectrum obtained from Fourier analysis, for example, will contain spurious power readings and energy spreading that actually represents nonlinearities in the data. This is because nonlinearities in the data will be represented within the Fourier power spectrum as higher-order harmonics since the transform itself utilizes a superposition of trigonometric functions. However, once the transform has been implemented, it is difficult to distinguish true power-frequency readings from spurious energy spreading due to nonlinearities inherent within the system. Furthermore, Fourier transform methods require piecewise stationarity of the time series under study. Thus any time-varying frequency content (usually present within EEG data) will be averaged out in the power spectrum. Although calculation of Fourier spectrograms has been proposed as a means to detect non-stationarity, this method still relies on linearity of the time series. Ideally, a decomposition method would be capable of extracting underlying oscillators from a seizure signal without any assumptions of the underlying waveform or characteristic time-scales of the oscillators. Recently, Huang and collaborators introduced EMD, a method for the extraction of (potentially non-linear and non-stationary) oscillators from any time series in an adaptive fashion [11].

The EMD can be characterized as an adaptive, data driven decomposition that results in a series of intrinsic mode functions (IMFs) that together comprise the underlying oscillations (or basis functions) within a dataset. The basis functions are determined from the dynamics of the signal itself which may be non-linear and/or non-stationary. Any time series may be represented as a linear combination of oscillators:

$$
\mathrm{x}(\mathrm{t})=\sum_{\mathrm{j}=1}^{\mathrm{N}} \mathrm{a}_{\mathrm{j}} \phi_{\mathrm{j}}(\mathrm{t}) .
$$

In Fourier decomposition, for example, each $\phi_{\mathrm{j}}(\mathrm{t})$ is a sine or cosine with a corresponding Fourier coefficient, $a_{j}$, for each $\phi_{\mathrm{j}}(\mathrm{t})$. In order to improve the decomposition of a time series to account for non-stationarity, other decompositions such as wavelet analysis have been applied; however, while they allow for non-stationarity, the basis functions are, again, determined before the decomposition and thus may be inappropriate for nonlinear signals.

The EMD has been mainly applied to hydrologic [12], atmospheric [13] and oceanic [14] time series, but it is widely believed to be applicable to any time series. The method can be briefly outlined as follows: The decomposition begins by identifying all maxima and minima of the data set followed by interpolation between the extrema using a cubic spline to obtain a maximum envelope $\mathrm{e}_{\max }(\mathrm{t})$ and a minimum envelope $e_{\min }(t)$. Next, the average of the envelopes $m(t)=$ $\left[\mathrm{e}_{\max }(\mathrm{t})+\mathrm{e}_{\min }(\mathrm{t})\right] / 2$ is computed and subtracted from the original data $\mathrm{x}(\mathrm{t})$ to obtain a residual $\mathrm{r}(\mathrm{t})=\mathrm{x}(\mathrm{t})-\mathrm{m}(\mathrm{t})$. This process is then repeated on the residual in an iterative fashion to obtain a series of IMFs. Because this is a numerical approximation scheme, the procedure must be refined by sifting the data through iteration of the first few steps (prior to subtraction of the mean) to obtain a zero-mean amplitude- and frequency-modulated signal which may be called a "proper rotation" or an IMF. Practically, the sifting process is optimized according to the particular data-set by defining an appropriate stopping criterion using, for example, number of sifts [11] accumulated energy [15], or confidence limit criteria [16] After the sifting and iteration procedure is complete, one obtains a series of modes plus the "trend" that represents the remainder of the decomposition when further sifting will not result in a proper IMF.

\section{Eigenvalue Decomposition}

Once the highest energy IMFs are obtained from each channel within a data segment, it is desirable to determine the strength of the relationships between oscillators. To accomplish this, each IMF is treated as a row vector and compiled into an $\mathrm{m} \times \mathrm{n}$ matrix, where $\mathrm{m}$ is the number of IMFs obtained from the analysis and $\mathrm{n}$ is the length of the time series. This set of time series represent the significant oscillators decomposed from the original time series via EMD. Next, the mutual phase coherence is calculated [17] this quantity is defined as:

$$
\mathrm{R}_{\mathrm{jk}}=\sum \exp \left[\mathrm{i}\left(\phi_{\mathrm{j}}(\mathrm{t})-\phi_{\mathrm{k}}(\mathrm{t})\right]\right.
$$

where "exp" represents the exponential, " $i$ " is $\sqrt{ }-1$, and each $\phi(t)$ is the phase of the analytic signal sampled at each discrete time instant. The sum ranges from $\mathrm{j}, \mathrm{k}=1$ to $\mathrm{N}(\mathrm{j} \neq \mathrm{k})$. This matrix is then analyzed for its eigenvalues and eigenvectors. For any eigenvalue-eigenvector pair, a phase correlation value may be assigned as the strength of the connection of a given eigenvalue (unique for a given IMF) and an eigenvector (unique for the entire set of IMFs obtained from all channels). Furthermore, each eigenvalue is ordered, with the largest eigenvalue representing the most strongly correlated cluster with the participation of each oscillator in a given cluster quantified by the value of the eigenvector. Specifically, one can assign the strength of the connection (participation) between the phases of any two IMFs within a single group via the square root of the IMF-associated eigenvalue $\left(\lambda_{n}\right)$ multiplied by the component-wise square of the IMF channel group eigenvector $\left(v_{n}^{2}\right)$ :

$$
\sqrt{ }\left[\lambda_{n} v_{n}^{2}\right]
$$


For example, if a matrix of completely unsynchronized oscillators is analyzed in this way, one would obtain $\lambda_{1}=\lambda_{2}=$ $\ldots=\lambda_{\mathrm{n}}=1$ with $\mathrm{v}_{\mathrm{j}}=(0,0, \ldots, 0,1,0,0, \ldots, 0)$ and no clusters would be identified in equation (6). If a set of fully synchronized oscillators is analyzed, one would obtain a single eigenvalue, whose value is identical to the number of oscillators, $\mathrm{N}$, with $\mathrm{v}_{1}=(1, \ldots, 1)$ and a single cluster in equation (4). Finally, for a set of oscillators with varying levels of synchrony, one would obtain a set of (strictly positive) eigenvalues. Those eigenvalues above one are considered significant and the components of their eigenvectors identify participation in the corresponding cluster calculated via equation (4). This threshold is set because, if a set of uncorrelated eigenvalues becomes more correlated, any increase in the eigenvalue must be offset by a corresponding decrease in other eigenvalues (as the sum of the eigenvalues is conserved). The eigenvalue decomposition method as cluster analysis is introduced and described in detail in ref. [8].

\section{RESULTS}

Depth recordings of brain activity were made from the CA3 region of the hippocampus bilaterally as well as in the anteromedial thalamus (targeted stereotactically) and over the surface of one hemisphere (contralateral to the hippocampus focally injected with kainic acid) in twelve rats. Both baseline and induced seizure activity was recorded for subsequent analysis. Figure 1 shows a ten-second sample for ictal activity. Visual inspection suggests a high degree of synchrony between all four electrode sites. Furthermore, the signals originating in both hippocampi appear more similar in waveform to one another than to anteromedial thalamus or the subdural signal. It should be noted that baseline activity represents brain activity in an anesthetized rat, as these are all acute in vivo studies. Thus, recordings made in these anesthetized animals show some level of pre-seizure synchrony most likely due to the activity of the anesthetic on the brain.

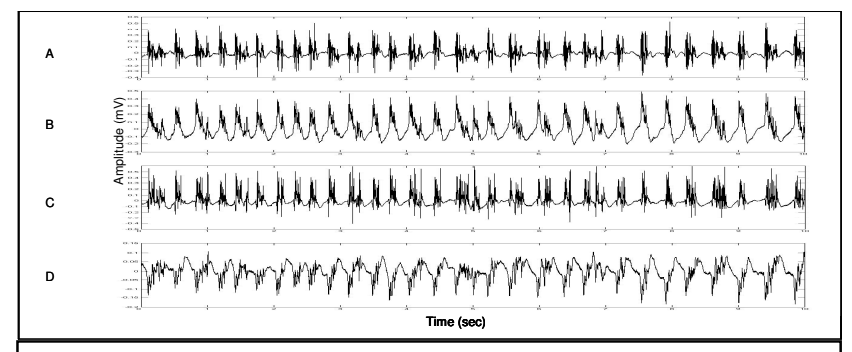

Figure 1: 10 second ictal sample. A: Focal Hippocampus. B:

Anteromedial Thalamus. C: Contralateral Hippocampus. D: Subdural recording over contralateral hemisphere.

The Hilbert analytic signal was constructed for each IMF in order to calculate the instantaneous phase for all selected IMFs. Then, the mean phase coherence was calculated for the entire matrix of phase values from each ten second sample. Finally, the eigenvalue decomposition was performed on this new mean phase coherence matrix to identify significantly synchronized clusters. A summary of all values of the participation index for these samples is shown in Figure 2 for the ictal samples corresponding to those in Figure 1. The red box indicates those clusters that are identified as significant (eigenvalues greater than 1). In general, interictal and baseline cluster analyses reveal fewer clusters than the same analysis for seizure samples. The interictal sample revealed four significant clusters while the ictal sample showed six. Furthermore, the frequencies of the oscillators participating in each cluster (as measured by maximal participation index value) were similar in the pre-ictal and interictal samples (data not shown).

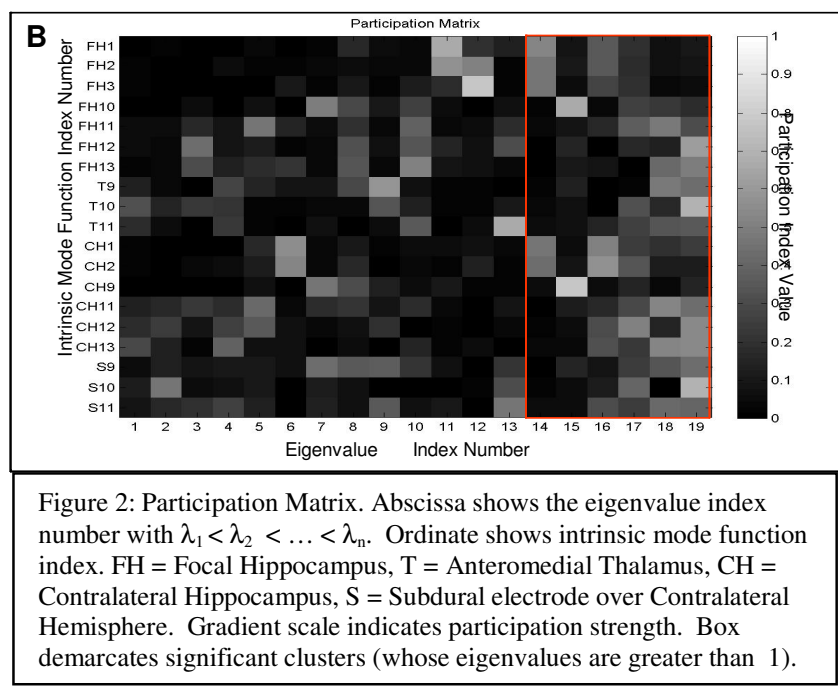

In order to highlight the instantaneous synchronous relationship between oscillators participating in a given cluster, the difference between the phases for each oscillator pair in the most significant cluster (highest eigenvalue) was calculated and plotted in Figure 3. These plots demonstrate the phase dynamics of the entire cluster. Because these oscillators are noisy, there are a number of phase slips (i.e. a change in the phase difference of $2 \pi$ ) thus the absolute value of phase difference is not relevant. However, those areas where the phase difference remains approximately constant represent regions of synchronization. Several of these areas of phase synchronization are highlighted in the figure by parentheses. Oscillators originating from the subdural electrode were not included in this analysis because these signals represent a summation of all activity within the (large) hemispheric dipole. Therefore, much of the activity recorded on that channel is contained within recordings made from subcortical sites. Thus, any synchrony between these oscillators and those obtained from depth sites is likely to be spurious because there will be high synchrony between identical oscillators. The ictal sample shows not only more oscillators, but also more regions of phase synchrony than the interictal samples. Furthermore, the phase synchrony is not constant between any two brain areas. Instead, the synchrony appears between different brain nuclei over time in an apparently reverberating circuit. 


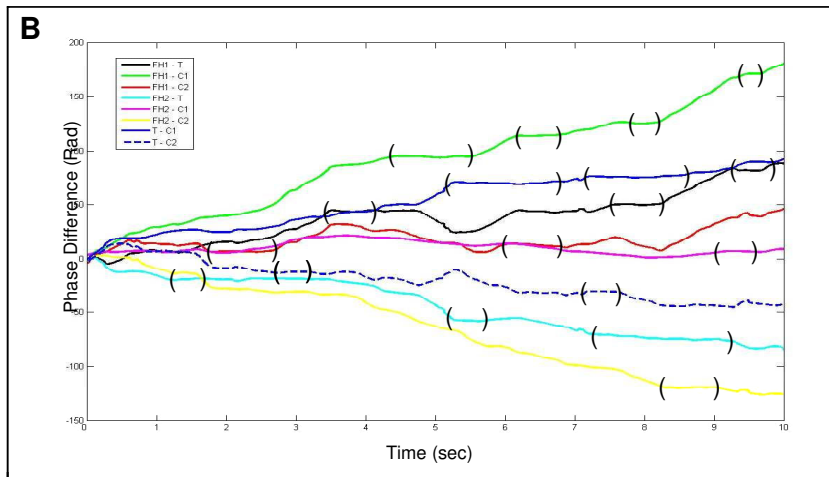

Figure 3: Largest Participation Cluster Phase Differences. This cluster contained two focal hippocampus (FH) IMFs, one thalamus (T) IMF, and two contralateral hippocampus (CH) IMFs.. Areas where the phase difference is constant parentheses) represent synchronous epochs.

\section{DisCUSSION}

Synchronized activity is implicated at all levels of neural activity in both pathological and normal states. It may be that both long- and short-range synchronization throughout the brain is a normal component of sensory and cognitive processing and that pathological states represent a derangement of this normal synchronization behavior. However, in order to characterize this synchrony in either pathological or normal states, it is necessary first to define a measure that is capable of detecting any form of synchrony that may arise. It is suggested here that prior filtering of a time series by a means that presupposes the underlying waveform and/or frequency content of the signal may eliminate important features of the signals underlying phase synchrony dynamics. Thus, it is proposed that a dynamic filter such as empirical mode decomposition is first employed in order to isolate any underlying oscillators. However, one important consideration in utilizing this technique is whether these intrinsic mode functions represent actual physiological oscillators or merely depict the phase dynamics of one or more physiological oscillators. This limitation is unlikely to interfere with synchrony analysis, but it may cause problems with direct physical interpretation of the oscillators obtained from the decomposition. Nevertheless, no other decomposition method to our knowledge is capable of yielding true physical oscillators at this point as all of them rely on some assumption as to the oscillator waveforms underlying the signal and usually involve fitting the time series to some a priori determined wave (e.g. a sine wave).

The analysis presented here is proposed as a means to determine the fine structure of phase dynamics during seizure activity in the brain. However, this method may be utilized to decompose and analyze phase dynamics in any time series thought to have multiple underlying oscillators contributing to nonstationary spatiotemporal phase dynamics.

\section{ACKNOWLEDGMENT}

We would like to thank Yue $\mathrm{Li}$ for his assistance in developing the software for on-line recording and analysis of seizure signals in the rat.

\section{REFERENCES}

[1] P. R. Roelfsema, A. K. Engel, P. König, and W. Singer, "Visuomotor integration is associated with zero time-lag synchronization among cortical areas," Nature, vol. 385, pp. 157-161, Janurary 1997.

[2] R. Levy, W. D. Hutchison, A. M. Lozano, and J. O. Dostrovsky, "Highfrequency synchronization of neuronal activity in the subthalamic nucleus of parkinsonian patients with limb tremor," J. Neurosci., vol. 20, pp. 7766-7775, October 2000

[3] H. W. Berendse, and C.J. Stam, "Stage-dependet patterns of disturbed neural synchrony in Parkinson's disease," Parkinsonianism Relat. Disord., vol. 13 suppl. 3, pp. S440-445, 2007.

[4] J. Gallinat, G. Winterer, C. S. Hermann, and D. Senkowski, "Reduced oscllatory gamma-band responses in unmedicated schizophrenic patients indicate impaired frontal network processing," Clin. Neurophys., vol. 115, pp. 1863-1874, Augusy 2004

[5] J. Jeong, "EEG dynamics in patients with Alzheimer's disease," Clin. Neurophys., vol. 115, pp. 1490-1505, 2004.

[6] A. V. Medvedev, "Epileptiform spikes desynchronize and diminish fast (gamma) activity of the brain. An 'anti-binding' mechanism?," Brain Res. Bull., vol. 58, pp. 115-128, May 2002.

[7] M. Le Van Quyen, V. Navarro, J. Martinerie, M. Baulac, and F. J. Varela, "Toward a neurodynamical unerstanding of ictogenesis," Epilepsia, vol. 44 suppl. 12, pp. 30-43, 2003.

[8] C. Allefeld, M. Müller, and J. Kurths, "Eigenvalue decomposition as a generalized synchronization cluster analysis," Inter. J. Bifurcation and Chaos, vol. 17, pp. 3493-3497, 2007.

[9] S. Bialonski, and K. Lehnertz, "Identifying phase synchronization clusters in spatially extended dynamical systems," Phys. Rev. E Stat. Nonlin. Soft Matter Phys., vol. 74, pp. 051909, November 2006.

[10] G. Paxinos, and C. Watson, The Rat Brain in Stereotaxic Coordinates, Academic Press, 2004

[11] N. E. Huan, Z. Shen, S. R. Long, M. C. Wu, H. H. Shih, Q. Zheng, N.C. Yen, C. C. Tung, and H. H. Liu, "The empirical mode decomposition and Hilbert spectrum for nonlinear and non-stationary time series analysis," Proc. R. Soc. Lond. A, vol. 454, pp. 903-995, 1998.

[12] K. I. Molla, M. S. Rahman, A. Sumi, and P. Banik, "Empirical mode decomposition analysis of climate changes with special reference to rainfall data," Discrete Dynamics in Nature and Society, vol. 2006, pp. 1-17, 2006.

[13] J. I. Salisbury, and M. Wimbush, "Using modern time series analysis techniques to predict ENSO events from the SOI time series," Nonlinear Processes in Geophysics, vol. 9, pp. 341-345, 2002.

[14] M. Dätig, and T. Schlurmann, "Performance and limitations of the Hilbert-Huang transformation (HHT) with an application to irregular water waves," Ocean Engineering, vol. 31, pp. 1783-1834, 2004.

[15] Y. Chen, and M. Q. Feng, "A technique to improve the empirical mode decomposition in the Hilber-Huang transform," Earthquake Engineering and Engineering Vibration, vol. 2, pp. 75-85, 2003.

[16] N. E. Huang, M. - L. C. Wu, S. R. Long, S. S. P. Shen, W. Qu, P. Gloersen, and K. L. Fan, "A confidence limit for the empirical mode decomposition and Hilbert spectral analysis," Proc. R. Soc. Lond. A, vol. 459, pp. 2317-2345, 2003.

[17] F. Mormann, T. Kreuz, R. G. Andrzejak, P. David, K. Lehnertz, and C. E. Elger, "Epileptic seizures are preceded by a decrease in synchronization,” Epilepsy Res., vol. 53, pp. 173-185, 2003. 